Physics

Physics Research Publications

\title{
GaMnAs-based hybrid multiferroic memory device
}

M. Overby

A. Chernyshov

L. P. Rokhinson
J. K. Furdyna 


\title{
GaMnAs-based hybrid multiferroic memory device
}

\author{
M. Overby, ${ }^{1}$ A. Chernyshov, ${ }^{1}$ L. P. Rokhinson, ${ }^{1, a)}$ X. Liu, ${ }^{2}$ and J. K. Furdyna ${ }^{2}$ \\ ${ }^{1}$ Department of Physics and Birck Nanotechnology Center, Purdue University, West Lafayette, \\ Indiana 47907, USA \\ ${ }^{2}$ Department of Physics, University of Notre Dame, Notre Dame, Indiana 46556, USA
}

(Received 29 February 2008; accepted 6 April 2008; published online 12 May 2008)

\begin{abstract}
We report a nonvolatile hybrid multiferroic memory cell with electrostatic control of magnetization based on strain-coupled GaMnAs ferromagnetic semiconductor and a piezoelectric material. We use the crystalline anisotropy of GaMnAs to store information in the orientation of the magnetization along one of the two easy axes, which is monitored via transverse anisotropic magnetoresistance. The magnetization orientation is switched by applying voltage to the piezoelectric material and tuning magnetic anisotropy of GaMnAs via the resulting stress field. (C) 2008 American Institute of Physics. [DOI: 10.1063/1.2917481]
\end{abstract}

A rapidly developing field of spintronics is based on the premise that substituting charge with spin as a carrier of information can lead to devices with lower power consumption, nonvolatility, and high operational speed. ${ }^{1-3}$ Despite efficient magnetization detection, ${ }^{4-6}$ magnetization manipulation is primarily performed by current-generated local magnetic fields and is very inefficient. The major weakness of current magnetoresistive random access memory implementations lies in the inherently nonlocal character of magnetic fields used to flip ferromagnetic domains during the write operation. Neither thermal ${ }^{7}$ nor current induced switching ${ }^{8}$ can completely solve the problem.

An attractive alternative to conventional ferromagnets is multiferroic materials, ${ }^{9}$ where ferromagnetic and ferroelectric properties coexist and magnetization can be electrostatically controlled via magnetoelectric coupling. In singlephase and mixed-phase multiferroics, ferromagnetic material must be insulating in order to avoid short circuits. Alternatively, magnetoelectric coupling can be indirectly introduced between ferromagnetic and ferroelectric materials via strain, and in this case, the ferromagnetic material can be conducting. A conceptual memory device made of ferromagnetic and piezoelectric materials has been proposed, ${ }^{10}$ and permeability changes in magnetostrictive films, ${ }^{11}$ changes in coercive field, ${ }^{12}$ and reorientation of the easy axis from in plane to out of plane in $\mathrm{Pd} / \mathrm{CoPd} / \mathrm{Pd}$ trilayers ${ }^{13}$ have recently been demonstrated.

Some ferromagnetic materials have a complex anisotropic magnetocrystalline energy surface and several easy axes of magnetization. If the energy barrier between adjacent easy-axis states can be controlled by strain, then a multiferroic nonvolatile multistate memory device can be realized. Magnetic anisotropy of dilute magnetic semiconductor GaMnAs films is largely controlled by epitaxial strain, ${ }^{14,15}$ with compressive (tensile) strain inducing in-plane (out-ofplane) orientation of magnetization. In GaMnAs compressively strained to (001) GaAs, there are two equivalent easy axes of in-plane magnetization: along the [100] and along the [010] crystallographic directions. Lithographically induced unidirectional lateral relaxation can be used to select the easy axis. ${ }^{16,17}$ In addition to the large in-plane crystalline anisotropy, there is a uniaxial anisotropy between the [110] and

\footnotetext{
${ }^{a)}$ Electronic mail: leonid@ @urdue.edu.
}

the $[1 \overline{10}]$ directions which is probably due to the underlying anisotropy of the reconstructed GaAs surface. ${ }^{18}$ We use this magnetocrystalline anisotropy in combination to the magnetostrictive effect to demonstrate a bistable memory device.

Molecular beam epitaxy at $265^{\circ} \mathrm{C}$ was employed to grow $15 \mathrm{~nm}$ thick epilayers of $\mathrm{Ga}_{0.92} \mathrm{Mn}_{0.08} \mathrm{As}$ on semiinsulating (001) GaAs substrates. The wafers were subsequently annealed for $1 \mathrm{~h}$ at $280^{\circ} \mathrm{C}$ in a nitrogen atmosphere, increasing the Curie temperature to $T_{C} \sim 80 \mathrm{~K}$ and reducing the cubic anisotropy. The GaMnAs layer was patterned into $2 \mu \mathrm{m}$ wide Hall bars oriented along the [110] axis by combination of e-beam lithography and wet etching, see Fig. 1. After lithography, $3 \times 3 \mathrm{~mm}^{2}$ samples were mechanically thinned to $\sim 100 \mu \mathrm{m}$ and attached to a multilayer piezoelectric lead zirconium titanate (PZT) ceramic with epoxy, aligning the [010] axis with the axis of polarization of the PZT. Application of positive (negative) voltage $V_{\mathrm{PZT}}$ across the piezoelectric introduces tensile (compressive) strain in the sample along the [010] direction, and strain with the opposite sign along the [100] direction proportional to the piezoelectric strain coefficients $d_{33} \approx-2 d_{31}$. Both coefficients decrease by a factor of 15 between room temperature and $4.2 \mathrm{~K}$. The induced strain $\varepsilon=\Delta L / L$ for both the [010] and the [100] directions was monitored with a biaxial strain gauge glued to the bottom of the piezoelectric measured in the Wheatstone bridge configuration: $\Delta \varepsilon=\varepsilon_{[010]}-\varepsilon_{[100]}$ $=(\Delta L / L)_{[010]}-(\Delta L / L)_{[100]}=\alpha\left(R_{[010]}-R_{[100]}\right) / R$, where $\alpha$ is the gauge sensitivity coefficient and $R$ is the resistance of the

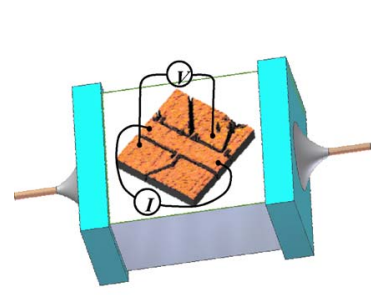

(a)

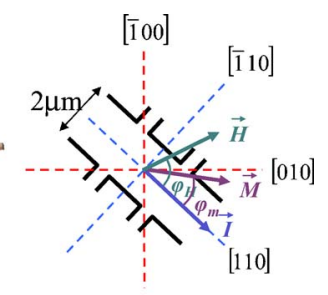

(b)
FIG. 1. (Color online) (a) Atomic force microscope image of a $2 \mu \mathrm{m}$ wide Hall bar attached to a piezoelectric. Strain is applied along the [100] and the [010] directions, red dashed lines on the sketch. (b) Hall bar with relative orientation of electrical current $\vec{I}$, magnetic field $\vec{H}$, and magnetization $\vec{M}$. 


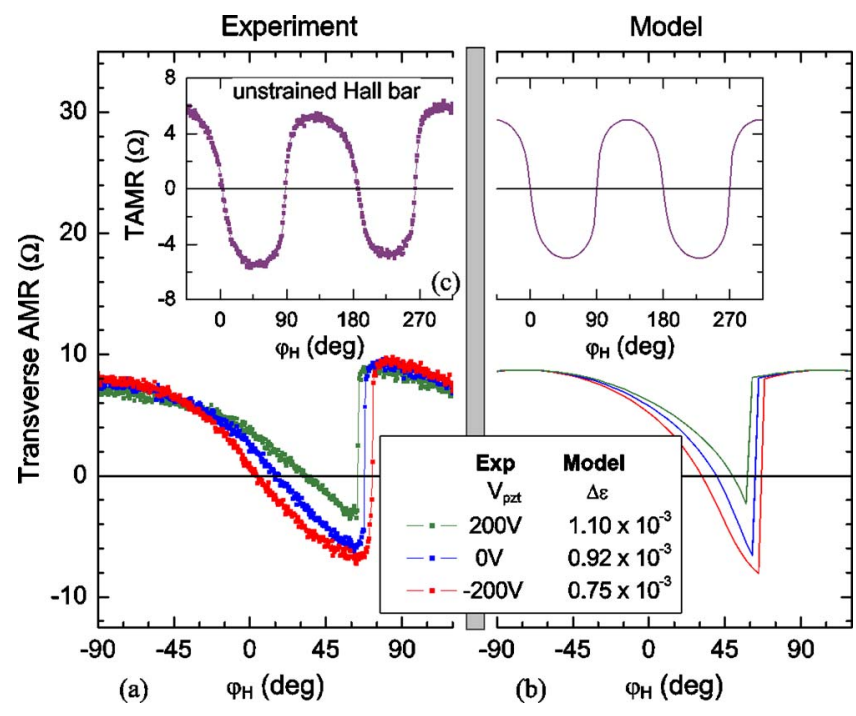

FIG. 2. (Color online) Experimentally measured (a) and modeled (b) transverse AMRs for the strained sample at different voltages on the piezoelectric [data for an unstrained sample are shown in (c)]. All data taken at $T=35 \mathrm{~K}$ and $H=50 \mathrm{mT}$. The model uses measured strain $\Delta \varepsilon$.

unstrained gauge. It has been shown before ${ }^{19}$ that the strain gradient across the piezoelectric and the sample is negligible: i.e., gauges glued on top of the sample and on the opposite side of the piezoelectric measure similar strain.

Direction of the in-plane magnetization $\vec{M}$ is measured via transverse anisotropic magnetoresistance (TrAMR), also known as giant planar Hall effect, ${ }^{20}$

$$
\operatorname{TrAMR}=\left(\rho_{\|}-\rho_{\perp}\right) \sin \varphi_{m} \cos \varphi_{m},
$$

where $\rho_{\|}$and $\rho_{\perp}$ are the resistivities for magnetization oriented parallel and perpendicular to the current. The sign and magnitude of TrAMR depend on the angle $\varphi_{m}$ between magnetization $\vec{M}$ and current $\vec{I} \|[110]$, see schematic in Fig. 1 . TrAMR reaches minimum (maximum) when $\vec{M} \|[010]$ $(\vec{M} \|[\overline{1} 00])$. Longitudinal AMR is $\propto \cos ^{2} \varphi_{m}$ and is not sensitive to the magnetization switching between $45^{\circ}$ and $135^{\circ}$.

In Fig. 2, TrAMR is plotted for the strained and unstrained Hall bars as magnetic field of constant magnitude $H=50 \mathrm{mT}$ is rotated in the plane of the sample. For the unstrained sample (not attached to the piezoelectric), there are four extrema for the field angle $\varphi_{H}$ around $45^{\circ}, 135^{\circ}, 225^{\circ}$, and $315^{\circ}$ with four switchings of magnetization by $90^{\circ}$ near the $[110]$ and the $[\overline{100}]$ directions. For the strained sample there is a gradual change in the angle of magnetization with only two abrupt $90^{\circ}$ switchings per field rotation, which indicates strong uniaxial anisotropy due to a highly anisotropic thermal expansion coefficient of the PZT stack $(+1 \mathrm{ppm} / \mathrm{K}$ along and $-3 \mathrm{ppm} / \mathrm{K}$ perpendicular to the piezoelectric stack).

Application of $H=50 \mathrm{mT}$ oriented at $\varphi_{H}=62^{\circ}$ compensates the thermally induced uniaxial strain and restores the original degeneracy between the [010] and the [100] magnetization directions of the unstrained GaMnAs for $V_{\mathrm{PZT}} \approx 0$. An additional strain is then applied by varying voltage on the piezoelectric. In Fig. 3, TrAMR is plotted as a function of measured strain $\Delta \varepsilon$, the corresponding $V_{\mathrm{PZT}}$ are approximately marked on the top axis (there is a small hysteresis in $\Delta \varepsilon$ versus $\left.V_{\mathrm{PZT}}\right)$. As additional compressive strain is applied Downloaded 03 Sep 2008 to 128.210.124.90. Redistribution subject

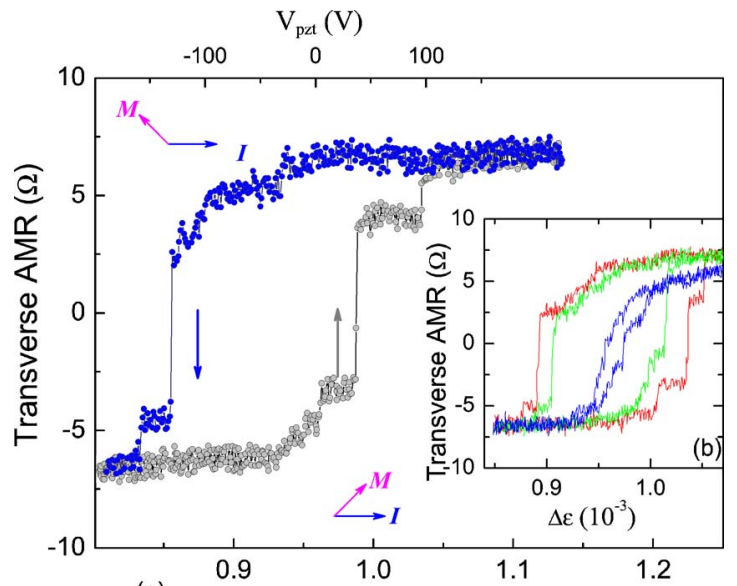

(a)

$$
\Delta \varepsilon\left(10^{-3}\right)
$$

FIG. 3. (Color online) (a) Transverse AMR (TrAMR) for the strained Hall bar as a function of uniaxial strain measured by a strain gauge. Static magnetic field $H=50 \mathrm{mT}$ is applied at $\varphi_{H}=62^{\circ}$, the data are taken at $T=35 \mathrm{~K}$. On the top axis approximate voltage on the piezoelectric is indicated. Orientation of magnetization relative to the current flow is shown schematically for the $\operatorname{TrAMR}>0$ and $\operatorname{TrAMR}<0$ states. (b) TrAMR is plotted for $H=50 \mathrm{mT}$ (red), $70 \mathrm{mT}$ (green) and $100 \mathrm{mT}$ (blue) measured at $T=25 \mathrm{~K}$.

along [010], this direction becomes the easy axis of magnetization and magnetization aligns itself with [010]. As additional tensile strain is applied along the [010] direction, the [100] direction becomes the easy axis and polarization switches by $90^{\circ}$. The switching occurs in a few steps, indicating a few-domain composition of our device. At $V_{\mathrm{PZT}}=0$, the magnetization has two stable orientations, $\vec{M} \|[\overline{1} 00]$ and $\vec{M} \|[010]$, and the orientation can be switched by applying a negative or a positive voltage on the piezoelectric. Thus, the device performs as a bistable nonvolatile magnetic memory cell with electrostatic control of the state.

The center of the loop can be shifted by adjusting $\varphi_{H}$ : e.g., a $1^{\circ}$ change shifts the center of the loop by $\Delta \varepsilon \approx 3.5 \times 10^{-5}$. As $H$ increases, the size of the hysteresis loop decreases, and the hysteresis vanishes for $H>100 \mathrm{mT}$; see inset in Fig. 3. At $H<40 \mathrm{mT}$, the loop increases beyond the $\pm 200 \mathrm{~V}$.

We model the strain along the [100] and the [010] directions as an extra magnetostatic energy density term $2 \varepsilon_{[100]} K_{\varepsilon} \sin ^{2}\left(\varphi_{m}+45^{\circ}\right)+2 \varepsilon_{[010]} K_{\varepsilon} \sin ^{2}\left(\varphi_{m}-45^{\circ}\right)$ $=\Delta \varepsilon K_{\varepsilon} \sin \left(2 \varphi_{m}\right)+$ const. Then, for a single domain magnet, the free energy density can be written as

$$
\begin{aligned}
E= & K_{u} \sin ^{2}\left(\varphi_{m}\right)+K_{1} / 4 \cos ^{2}\left(2 \varphi_{m}\right)+H M \cos \left(\varphi_{m}-\varphi_{H}\right) \\
& +\Delta \varepsilon K_{\varepsilon} \sin \left(2 \varphi_{m}\right),
\end{aligned}
$$

omitting constant offset, where $K_{1}, K_{u}$ and $K_{\varepsilon}$, are cubic, uniaxial, and strain anisotropy constants; $H$ is the applied in-plane magnetic field; and $\varphi_{m}$ and $\varphi_{H}$ are the angles between the [110] direction and magnetization and magnetic field respectively; see schematic in Fig. 1 . We assume that $K_{\varepsilon}$ is the same for the [100] and the [010] directions.

In equilibrium, the magnetization orientation $\varphi_{m}$ minimizes the free energy, $d E / d \varphi_{m}=0$ and $d^{2} E / d \varphi_{m}^{2}>0$. The TrAMR can be calculated from Eq. (1) for a given angle $\varphi_{H}$ of the external field $H$. From the fits to the experimental TrAMR data, we can extract the anisotropy constants $K_{1}, K_{u}$, and $K_{\varepsilon}$. The model captures all the essential features of the to AIP license or copyright; see http://apl.aip.org/apl/copyright.jsp 

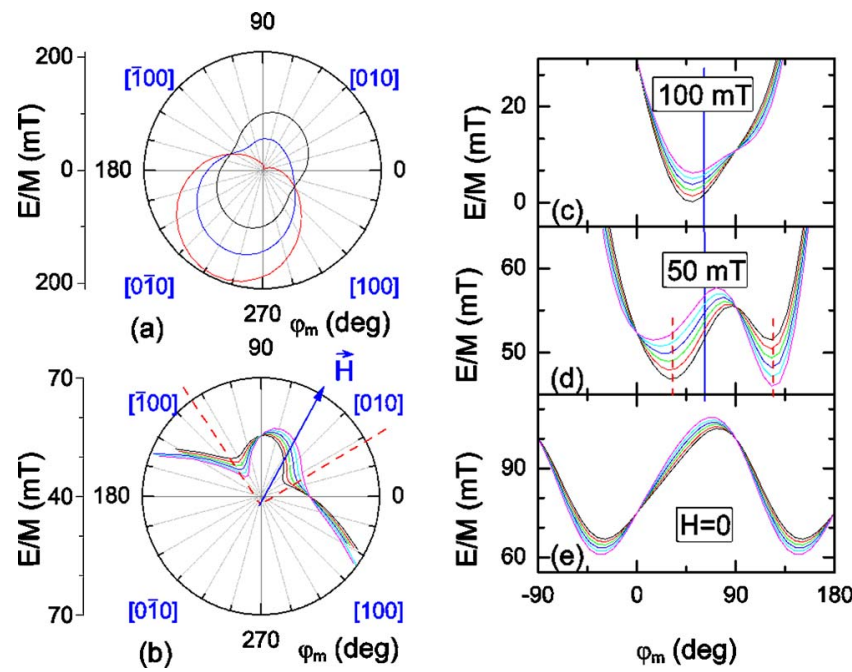

FIG. 4. (Color online) (a) Polar plot of magnetostatic energy $E / M$ as a function of magnetization angle $\varphi_{m}$ for $H=0$ (black), $50 \mathrm{mT}$ (blue) and $100 \mathrm{mT}$ (red) for $\varphi_{H}=62^{\circ}$ and $V_{\mathrm{PZT}}=0\left(\Delta \varepsilon K_{\varepsilon}=16 \mathrm{mT}\right)$. [(b)-(d)] Angular dependence of $E / M$ for $\Delta \varepsilon K_{\varepsilon}=13-19 \mathrm{mT}$ (black to magenta) for $H=0$ (e), $H=50 \mathrm{mT}[(\mathrm{b})$ and (d)], and $H=100 \mathrm{mT}$ (c). Blue line and arrow mark $\varphi_{H}=62^{\circ}$; dashed red lines indicate two stable orientations of magnetization.

data, and corresponding fits are shown in Fig. 2 for the strained and unstrained devices. For the unstrained device anisotropy fields $2 K_{1} / M=40 \mathrm{mT}$ and $2 K_{u} / M=6 \mathrm{mT}$. These values are significantly smaller than the previously reported values for as-grown (not annealed) wafers. ${ }^{20,21}$ For the sample attached to the piezoelectric the crystalline anisotropy field remains the same, but the uniaxial anisotropy increases to $2 K_{u} / M=50 \mathrm{mT}$. The strain-induced anisotropy field $\Delta \varepsilon K_{\varepsilon} / M$ varies between 13 and $19 \mathrm{mT}$ for different $V_{\text {PZT }}$ between -200 and $200 \mathrm{~V}$, the coefficient $K_{\varepsilon} / M=17 \mathrm{~T}$.

To illustrate the mechanism of magnetization switching, we plot magnetic energy density normalized by magnetization $E / M$ [Eq. (2)] as a function of $\varphi_{m}$ in Fig. 4. At $H=0$, there are only two minima along the [100] axis due to the large uniaxial strain [see Figs. 4(a) and 4(e)], caused by anisotropic thermal expansion coefficient of the piezoelectric. With $H=50 \mathrm{mT}$ applied at $\varphi_{H}=62^{\circ} \mathrm{E} / \mathrm{M}$ has two minima: at $\varphi_{m}=32^{\circ}$ and at $123^{\circ}$, i.e., close to the [010] and to the [100] crystallographic directions. For the strain field $\Delta \varepsilon K_{\varepsilon} / M=13 \mathrm{mT}$ the global minimum is at $\varphi_{m}=32^{\circ}$, and in equilibrium the magnetization is oriented along the [010] direction. As the strain field increases to $19 \mathrm{mT}$, the two minima switch, and $\varphi_{m}=123^{\circ}$ becomes the global minimum. It is interesting to note that there is always a small barrier between the two minima. Unless the barrier is an artifact of our model, the switching of magnetization should be either temperature activated or should occur via macroscopic quantum tunneling.

In our experiments, the magnetic field balances the residual strain due to anisotropic thermal expansion of the PZT. Alternatively, intrinsic piezoelectric properties of GaAs can be utilized. In this case, there will be no thermally induced strain and electrostatic switching of the magnetization direction can be realized without applying an external compensating magnetic field. Scaling of the piezoelectric element from $0.5 \mathrm{~mm}$ down to $\sim 1 \mu \mathrm{m}$ will compensate for a small strain coefficient in $\mathrm{GaAs}\left(d_{33}^{\mathrm{PZT}} \approx 10 d_{14}^{\mathrm{GaAs}}\right.$ at $\left.30 \mathrm{~K}\right)$, reduce operating voltage to a few volts, and allow electrostatic control of individual memory cells.

The work was supported by NSF under the Grant Nos. ECS-0348289 (Purdue) and DMR-0603752 (Notre Dame).

${ }^{1}$ H. Ohno, Science 281, 951 (1998).

${ }^{2}$ G. A. Prinz, Science 282, 1660 (1998).

${ }^{3}$ S. A. Wolf, D. D. Awschalom, R. A. Buhrman, J. M. Daughton, S. V. Molnar, M. L. Roukes, A. Y. Chtchelkanova, and D. M. Treger, Science 294, 1488 (2001).

${ }^{4}$ M. Baibich, J. Broto, A. Fert, F. Nguyen Van Dau, F. Petroff, P. Etienne, G. Creuzet, A. Friederich, and J. Chazelas Phys. Rev. Lett. 61, 2472 (1988).

${ }^{5}$ G. Binasch, P. Grünberg, F. Saurenbach, and W. Zinn, Phys. Rev. B 39, 4828 (1989).

${ }^{6}$ J. Moodera, L. Kinder, T. Wong, and R. Meservey Phys. Rev. Lett. 74, 3273 (1995)

${ }^{7}$ M. Kerekes, R. Sousa, I. Prejbeanu, O. Redon, U. Ebels, C. Baraduc, B. Dieny, J.-P. Nozieres, P. Freitas, and P. Xavier, J. Appl. Phys. 97, 10 (2005).

${ }^{8}$ E. Myers, D. Ralph, J. Katine, R. Louie, and R. Buhrman, Science 285, 867 (1999).

${ }^{9}$ W. Eerenstein, N. Mathur, and J. Scott, Nature (London) 442, 759 (2006).

${ }^{10}$ V. Novosad, Y. Otani, A. Ohsawa, S. Kim, K. Fukamichi, J. Koike, K.

Maruyama, O. Kitakami, and Y. Shimada, J. Appl. Phys. 87, 6400 (2000).

${ }^{11}$ K. Arai, C. Muranaka, and M. Yamaguchi, IEEE Trans. Magn. 30, 916 (1994).

${ }^{12}$ H. Boukari, C. Cavaco, W. Eyckmans, L. Lagae, and G. Borghs, J. Appl. Phys. 101, 054903 (2007).

${ }^{13}$ J.-W. Lee, S.-C. Shin, and S.-K. Kim, Appl. Phys. Lett. 82, 2458 (2003).

${ }^{14}$ U. Welp, V. K. Vlasko-Vlasov, X. Liu, J. K. Furdyna, and T. Wojtowicz, Phys. Rev. Lett. 90, 167206 (2003).

${ }^{15}$ X. Liu, Y. Sasaki, and J. K. Furdyna, Phys. Rev. B 67, 205204 (2003).

${ }^{16}$ S. Hümpfner, K. Pappert, J. Wenisch, K. Brunner, C. Gould, G. Schmidt, L. W. Molenkamp, M. Sawicki, and T. Dietl, Appl. Phys. Lett. 90, 102102 (2007).

${ }^{17}$ J. Wenisch, C. Gould, L. Ebel, J. Storz, K. Pappert, M. J. Schmidt, C. Kumpf, G. Schmidt, K. Brunner, and L. W. Molenkamp, Phys. Rev. Lett. 99, 077201 (2007).

${ }^{18}$ U. Welp, V. Vlasko-Vlasov, A. Menzel, H. You, X. Liu, J. Furdyna, and T. Wojtowicz, Appl. Phys. Lett. 85, 260 (2004).

${ }^{19}$ M. Shayegan, K. Karrai, Y. Shkolnikov, K. Vakili, E. De Poortere, and S. Manus, Appl. Phys. Lett. 83, 5235 (2003).

${ }^{20}$ H. Tang, R. Kawakami, D. Awschalom, and M. Roukes, Phys. Rev. Lett. 90, 107201 (2003).

${ }^{21}$ D. Y. Shin, S. J. Chung, S. Lee, X. Liu, and J. K. Furdyna, Phys. Rev. B 76, 035327 (2007). 